\title{
Trazador de curvas IV portátil para diagnóstico de centrales de generación fotovoltaica
}

Dr. Andrés D. Firman, Sr. Bertotto, Olivio Augusto, Dr. Luís H. Vera, Dr. Manuel Cáceres, Dr. Arturo J. Busso (*)

Palabras clave: Energía Solar fotovoltaica, instrumentación, caracterización eléctrica.

\section{Resumen}

En este artículo se describen los aspectos de diseño, la metodología constructiva principal y el desarrollo de un dispositivo trazador de curvas IV portable, para ser utilizado en centrales generadoras fotovoltaicas, con el fin de realizar caracterización y diagnóstico de los generadores que la integran. Los rangos máximos de funcionamiento del instrumento construido son 900 $\mathrm{V}$ de tensión en CC y hasta los $25 \mathrm{~A} \mathrm{CC}$ en corriente. Además, se presentan los resultados obtenidos en su caracterización, se indican las incertezas de los canales de medición por lo que se concluye que el equipo desarrollado cumple satisfactoriamente con la función con la que fue diseñado. El equipo además logró ser transferido a una empresa del medio local.

\section{Introducción}

Una de las formas más ampliamente difundidas para realizar controles de diagnóstico de dispositivos fotovoltaicos (FV) es mediante el trazado de la curva corriente-tensión (curva IV) del generador FV. Trabajando con la misma, se logra calcular los valores de los componentes del modelo equivalente eléctrico que lo representa, junto con los valores de sus parámetros térmicos. También se puede individualizar la potencia máxima que el generador FV es capaz de erogar en las condiciones de operación al momento del trazado.

Trabajar con la curva, permite obtener conclusiones directamente del análisis del perfil que presenta la misma, detectando problemas de generación concluidos en base a la deformación que existiera en la misma (Firman et al, 2013). La Fig. 1, indica la forma general de una curva IV, en la cual se distinguen los puntos principales

() Campus Deodoro Roca, Av. Libertad 5460

Contactos: afirman@ger-unne.com.ar, bertotto.olivio@gmail.com, 1vera@ger-unne.com.ar, @ger-unne.com.ar, 
que son la corriente de corto circuito Icc, la tensión de circuito abierto $V$ ca y el punto de máxima potencia Prn.

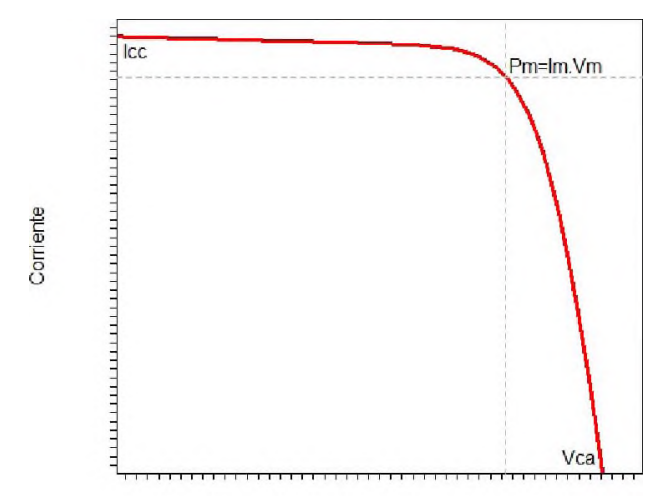

Tensión

Figura 1 - Perfil tipico de una curva IV de un dispositivo $F V$.

Para trazar la curva IV de un dispositivo $\mathrm{FV}$ se precisan un conjunto de valores de corriente y tensión desde el estado de cortocircuito al de circuito abierto, con suficientes valores que permitan una correcta representación de la curva a lo largo de los posibles estados de polarización. En la bibliografía se estima que se necesitan para ello, más de 100 puntos. Además el trazado a sol natural de la curva IV debe ser lo suficientemente veloz de manera de que las condiciones climáticas se consideren invariables durante todo el proceso, para ello se procura realizar el trazado de la curva en un intervalo de entre los $20 \mathrm{~ms}$ a alrededor de los 200 ms de duración, de manera de considerar constante (sobre todo en un día claro sin presencia de nubes y viento calmo), la irradiancia y la temperatura de célula FV que resultan críticas para el estudio
(Martínez et al, 2012) (Firman et al, 2010) (Muñoz y Lorenzo, 2006).

Hoy en día es común encontrar en funcionamiento, en la Argentina y en el resto del mundo, plantas generadoras $\mathrm{FV}$ o centrales FV cuyas ramas componentes oscilan entre los 400 a $900 \mathrm{~V}$ CC y corrientes comprendidas alrededor de los 10 A CC (Cáceres et al, 2018) (Martínez et al, 2012), lo que agrega un extra de dificultad al proceso de manipulación de la energía durante el trazado.

El trazado de la curva IV de un generador FV no se encuentra completo si al momento del ensayo no se registra, con una precisión aceptable, la irradiancia en el plano del generador y su temperatura de célula, para ello es común recurrir a celdas de irradiancia calibradas, que se comportan de igual manera, espectralmente y angularmente, que el generador (Firman et al, 2016). Para el caso de la medición de temperatura se utilizan comúnmente medidores indirectos basados en resistencias de platino (PT100), o bien, también indirectamente, mediante el comportamiento de la tensión de circuito abierto de una célula de referencia.

Para ello se necesita contar con la precisión electrónica comparable a dispositivos de medición comerciales, de manera de reducir las incertezas asociadas a cada uno de los parámetros a medir. Además, debe ser lo suficientemente robusto si se considera que el dispositivo debe ser portátil, ya que debe trasladarse a terreno, lo que dificulta aún más el desarrollo.

En el mercado actual, sobre todo en lo 
referente al trazado de curvas IV relacionado a generadores FV para sistemas conectados a red (SFCR), se popularizan los dispositivos que basan su funcionamiento en lo que se conoce como "carga del tipo capacitiva", la misma es capaz de polarizar en todo el rango deseado de trazado, adquiriendo datos durante el transitorio de carga de un banco de capacitores especialmente diseñado, conectado directamente al generador FV (Ghareeb et al, 2018).

En resumen, para el trazado de curvas se requiere de la medición precisa de cuatro parámetros, como ser la corriente, la tensión, la irradiancia solar y la temperatura de célula, además de la electrónica de medición y la electrónica de potencia adecuada para controlar todo el dispositivo.

Los trazadores comerciales no se encuentran en el mercado nacional y los de origen extranjero poseen un costo que los hace prohibitivo o bien que no se adecuan a los requerimientos locales. Por ello se contempló la necesidad de desarrollar dentro del entorno de la universidad un dispositivo capaz de trazar curvas IV, portátil y orientado a centrales FV, con los rangos de trabajo citados. Tarea en la que el Grupo en Energías Renovables viene desempeñándose desde hace más de 10 años, por lo que se cuenta con extendida experiencia en el área.

\section{Metodología}

En este apartado se describirán los aspectos fundamentales de diseño tenidos en cuenta para el desarrollo del trazador de curvas IV, para lo cual se proyecta, que debe ser portable, por lo que todo el conjunto debe caber dentro de una sola unidad. Un diagrama en bloques que integra todas las etapas se muestra en la Fig. 2.

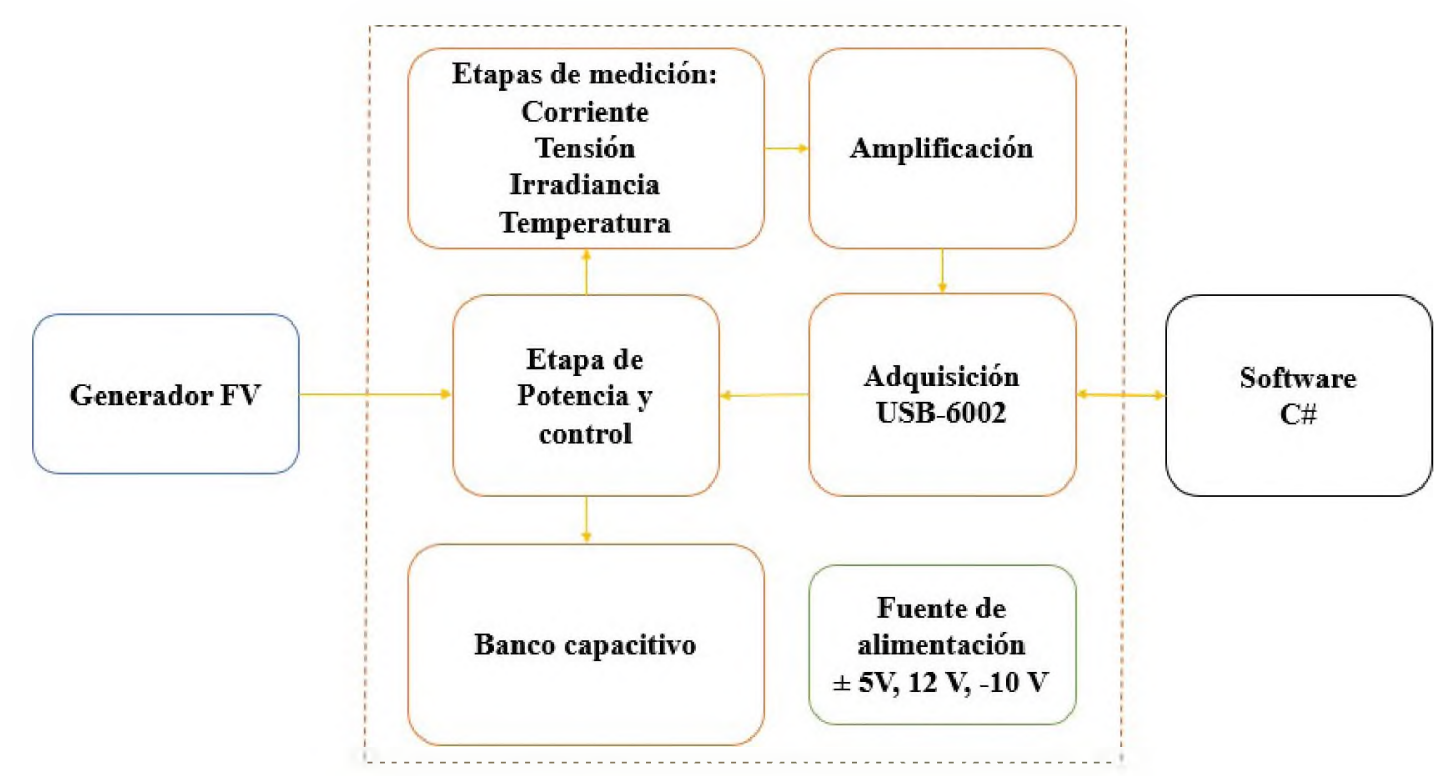

Figura 2 - Diagrama en bloques del trazador de curvas IV desarrollado 
Dentro de las etapas de medición se encuentra la etapa de medición de irradiancia, que se realiza mediante una celda de referencia especialmente desarrollada y calibrada en el mismo grupo de investigación (Firman et al, 2016), la etapa de medición de temperatura se realiza mediante un dispositivo PT100 por medio de una fuente de corriente constante.

La medición de tensión se realiza a cuatro hilos, de manera de eliminar influencia en la caída de los conductores de conexión. La alta tensión es reducida mediante un divisor resistivo de potencia adecuada y luego amplificada y dirigida a la etapa de medición.

La medición de corriente se realiza por medio de una resistencia del tipo shunt, también conectada a cuatro hilos, para ello se utilizó un elemento comercial de clase 0,5 de 25 A. Nuevamente, la señal proveniente del shunt se amplifica y se dirige a la etapa de medición.

Las señales son tratadas en la etapa de amplificación, en ella las ganancias necesarias para cada caso varían entre 1 a 100, para ello se recurrió a amplificadores de instrumentación AD620 de la empresa Analog Devices (AD620, 2018) de excelentes prestaciones. Se utilizaron en las etapas criticas resistencias de $0,1 \%$ de precisión y capacitores de filtrado de al menos del $1 \%$.

La etapa de potencia y control es controlada con una placa multifunción, NIUSB6002 (NI USB-6002, 2018), la cual es un dispositivo USB de múltiples entradas/ salidas, que cuenta con 8 canales de entrada analógicos de 16 bits, y capaz de tomar muestras con una tasa de hasta los $50 \mathrm{kS} / \mathrm{s}$.
Para la fuente de alimentación de todo el dispositivo se recurrió a una batería de plomo-gel de 2,3 Ah. A partir de ella, los voltajes secundarios necesarios en cada etapa se obtienen mediante fuentes lineales y fuentes del tipo switching. Esta última permite generar un voltaje negativo logrando obtener datos del segundo cuadrante en el trazado de la curva IV, con el fin de caracterizar con mayor precisión la corriente de cortocircuito del generador FV bajo ensayo.

El control de todo el sistema se realiza mediante un software especialmente desarrollado que coordina y supervisa el estado de todas las etapas mediante una lógica incorporada al mismo, de manera de hacerlo seguro tanto para el circuito de control como para el operario. Este se desarrolló en la plataforma Visual Studio 2015 y fue programado en lenguaje $\mathrm{C \#}$.

La etapa de potencia y control se realiza mediante dispositivos industriales de elevada confiabilidad controlados por una etapa opto-acoplada y para la carga se realizó la asociación serie paralelo de capacitores consiguiendo un banco de $2200 \mu \mathrm{F}$ y 900 V. En la construcción del banco capacitivo, se instalaron resistencias balanceadoras, de manera de distribuir uniformemente los voltajes sobre los mismos.

En definitiva, el equipo es capaz de trazar curvas IV de hasta $900 \mathrm{~V}$ y $25 \mathrm{~A}$, considerándose un rango adecuado para operar en las centrales de generación FV.

\section{Resultados}

El instrumento o dispositivo trazador de curvas IV desarrollado se visualiza en la 
Fig. 3a, en la misma se aprecia una única unidad en formato transportable tipo maletín con cavidades especialmente diseñadas para colocar una Tablet en la que se ejecuta el software de control, junto con otras destinadas a herramientas y a la celda de referencia utilizada. En la Fig. 3b, se muestra

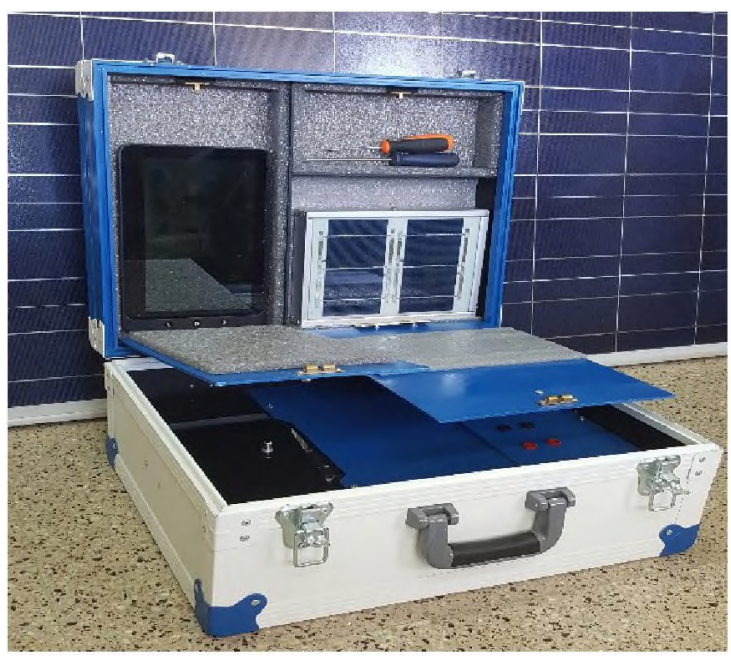

una curva IV tomada en el peor caso posible para el funcionamiento del equipo, que es el caso de un único módulo FV cuyo rango de operación es mucho menor que los de diseño, conduciendo a un transitorio de muy pocos milisegundos, donde puede apreciarse una correcta representación de la curva.

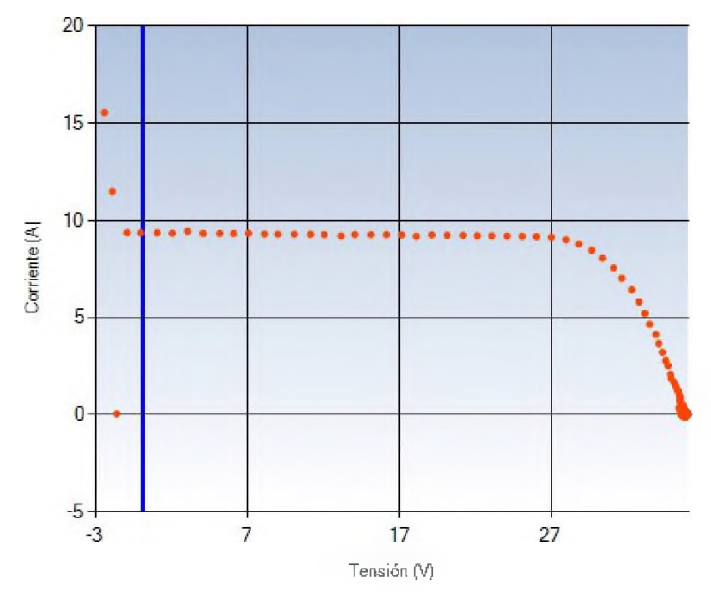

Figura 3 - a) Fotografia del instrumento construido. b) Captura de una curva obtenida con el instrumento desarrollado.

La incertezas de los canales de entrada se dividen en 3 grupos, la incertezas asociadas al transductor (shunt, divisor, celda, etc) las incertezas asociadas a las etapas amplificadoras y las incertezas asociadas a la conversión analógica de la placa de medición y control, que incluso estas últimas además dependen de la estabilidad de la fuente de alimentación. Por tal motivo se recurre a cálculos estadísticos, en donde se considera aceptable un intervalo de confianza del 95 $\%$ teniendo en cuenta la numerosa cantidad de puntos registrados en el trazado y el campo de operación del instrumento. Del ensayo a media escala se obtienen como resultados las siguientes incertezas para los canales de entrada;

Canal de corriente $\simeq 1,6 \%$

Canal de tensión $\simeq 0,6 \%$

Estas incertezas se consideran adecuadas para un instrumento de campo.

\section{Conclusiones}

Se logró efectivamente diseñar, construir y verificar las incertezas de un instrumento trazador de curvas IV destinado al diagnóstico de centrales fotovoltaicas con rangos máximos de medición que van desde los $900 \mathrm{~V}$ CC a los $25 \mathrm{~A} \mathrm{CC}$.

$\mathrm{E}$ l equipo desarrollado es portable, $\mathrm{pu}-$ diendo ser fácilmente trasladado hasta las regiones donde necesita ser empleado. 
Se verificó su funcionamiento y se determinaron las incertezas asociadas a los canales de corriente y tensión, los que se consideran satisfactorios para trabajo en campo. Esto se logra en base a la calidad de los componentes empleados en su cons-

\section{Bibliografía}

A. Firman, V. Toranzos, L. Vera, A. Busso, and J. de la Casa (2013). Passive monitoring of the power generated in grid connected PV systems. Energy Procedia. Elsevier.

F. Martínez-Moreno, E. Lorenzo, J. Muñoz, and R. Moretón (2012). On the testing of large PV arrays. Progress in photovoltaics: Research and Applications, 20(1):100-105, 2012

M. Cáceres, A. Busso, L. Vera, A. Firman (2018). First grid-tied photovoltaic system in notrhern argentina: characterization and analysis. Dyna. Energetic technology research article unconventional energy sources.

A. Firman, V. Toranzos, A. Busso, C. Cadena, and L. Vera (2010). Sistema híbrido para la caracterización eléctrica de arreglos fotovoltaicos. Avances en Energías Renovables y Medio Ambiente, 14.

J. Muñoz and E. Lorenzo. Capacitive load based trucción junto con estrategias de diseño adecuadas.

El equipo descrito se transfirió a una empresa del medio logrando realizar el vínculo entre empresas nacionales y la Universidad. on igbts for on-site characterization of PV arrays. Solar energy, 80(11):1489-1497, 2006

A. Firman, L. Vera, A. Busso. (2016). Fabricación y calibración de celdas de referencia para determinar la irradiancia efectiva en SFCR. VI Congresso Brasileiro de Energia Solar-Belo Horizonte.

A. Ghareeb, M. Tamimi, M. Jaber, S. Jaradat, \& T. Khatib. (2018). A new method for extracting IV characteristic curve for photovoltaic modules using artificial neural networks. In 20185 th International Conference on Electrical and Electronic Engineering (ICEEE) (pp. 473-476). IEEE.

AD620 (2018). Ad 620 Low Cost Low Power Instrumentation Amplifier, Analog Devices. Data Sheet.

NI USB-6002 (2018). Specifications NI USB6002 Low-Cost DAQ USB Device. National Instruments. Data Sheet. 